\title{
FATOR DE BIOCONCENTRAÇÃO DE POLUENTES ORGÂNICOS DE LODOS EM FRUTOS DE LARANJEIRAS
}

\author{
LOURIVAL COSTA PARAÍBA* \\ RITA CARLA BOEIRA** \\ CLÁUDIO MARTIN JONSSON** \\ JOSE MARÍA CARRASCO ${ }^{* * * *}$
}

\begin{abstract}
Estimou-se o fator de bioconcentração de dezenove poluentes orgânicos para verificar quais devem ser monitorados em frutos de laranjeiras, cultivadas em solos tratados com lodo proveniente de estações de tratamento de esgotos. O modelo Fruit Tree Model (FTM) foi aplicado em cultivo hipotético de laranjeiras, utilizando-se a massa molar, a pressão de vapor, a solubilidade em água e o coeficiente de partição octanol-água de poluentes (compostos orgânicos não-iônicos) que podem ser encontrados em lodos. Os coeficientes de sorção no carbono orgânico do solo, de partição caule-água e o fator de concentração no fluxo de transpiração foram calculados por meio de expressões que correlacionam cada um desses parâmetros com o coeficiente de partição octanol-água. Obtendo-se o fator de bioconcentração e a concentração do poluente no solo é possível estimar a quantidade desses agentes químicos ingeridos diariamente pelo consumo de frutas frescas. Valores limites de poluentes em lodos podem ser calculados a partir do fator de bioconcentração do poluente em frutas. Os poluentes indicados pela estimativa para monitoramento em frutos de laranjeiras cultivadas com lodo foram: 1,2-diclorobenzeno, 1,3diclorobenzeno, 1,4-diclorobenzeno, 2,4-dinitrofenol, 3,3-diclorobenzidina e nitrobenzeno.
\end{abstract}

PALAVRAS-CHAVE: FERTILIZANTE; CITRUS; INGESTÃO DIÁRIA; BIOSSÓLIDO; LODO.

* Doutor em Matemática Aplicada, Pesquisador da Embrapa Meio Ambiente, Jaguariúna, São Paulo, Brasil (e-mail: lourival@cnpma.embrapa.br).

** Doutora em Agronomia, Pesquisadora da Embrapa Meio Ambiente, Jaguariúna, São Paulo, Brasil (email: rcboeira@cnpma.embrapa.br).

*** Doutor em Biologia, Pesquisador da Embrapa Meio Ambiente, Jaguariúna, São Paulo, Brasil (e-mail: jonsson@cnpma.embrapa.br).

**** Doutor em Química, Professor Catedrático do Departamento de Biotecnologia da Universidade Politécnica de Valencia, Valencia, Espanha (e-mail: jmcarras@btc.upv.es). 


\section{INTRODUÇÃO}

O lodo é o resíduo obtido do processo de tratamento primário e secundário de águas servidas ou de esgotos. O conhecimento dos agentes químicos constituintes de lodos permite avaliar o risco de contaminação alimentar e ambiental decorrente da sua utilização como fertilizantes agrícolas. $O$ lodo, material de composição predominantemente orgânica, pode ser usado para melhorar a qualidade de solos agrícolas por meio da reciclagem de nutrientes (BETTIOL e SANTOS, 2001). No entanto, características desfavoráveis podem estar presentes nos lodos e limitar a quantidade a ser aplicada no solo. Em estudos realizados em plantações de laranjeiras, MADEJON et al. (2003) observaram que aplicações repetidas de quantidades moderadas de lodos promoveram efeitos positivos nas propriedades químicas e bioquímicas do solo, assim como na produção dos frutos.

No Brasil existem estudos de impactos ambientais de organismos patogênicos e de poluentes inorgânicos presentes em lodos, como metais pesados, ou gerados no solo após sua aplicação, como nitratos (BOEIRA, LIGO e DYNIA, 2002), mas com pouca informação disponível sobre compostos orgânicos poluentes (PARAÍBA e SAITO, 2005). Efeitos adversos em sistemas biológicos, tais como genotoxicidade e carcinogenecidade, têm sido atribuídos aos metais pesados (MIADOKOVA et al., 1999) e poluentes orgânicos encontrados em lodos (NEDELCHEVA et al., 1998).

Em decorrência da mistura de esgotos primários domésticos com industriais pode existir diversidade não desprezível de poluentes orgânicos em lodos (TSUTIYA, 2001). Isto porque as estações de tratamento de esgoto recebem grande espectro de moléculas orgânicas em esgotos domésticos ou industriais que não são completamente eliminadas durante o processo de tratamento (TERNES, 1998; TERNES et al., 1999). É possível encontrar em lodos inúmeros compostos de diferentes estruturas químicas, tais como antibióticos, anticoncepcionais, cosméticos, hormônios, azeites, detergentes, antiinflamatórios ou fármacos psiquiátricos (BOYD, 2003; CARGOUET et al., 2004; CARBALLA et al., 2004; CARBALLA et al., 2005; TERNES et al., 1999; TERNES, 1998).

ENGWALL e HJELM (2000) demonstraram que vegetais cultivados em solos nos quais foram adicionados lodos continham níveis de dioxinas impróprios para o consumo como alimento. ABAD et al. (2005) encontraram nonil fenol e nonil fenol etoxilado em lodos de estações de tratamento da Catalunha, Espanha, em níveis inadequados para serem utilizados como fertilizantes agrícolas. GAO e ZHU (2004) demonstraram que raízes e caules de plantas podem bioconcentrar fenantreno e pireno, dois poluentes orgânicos freqüentemente encontrados em lodos.

Segundo TSUTIYA (2001), os lodos produzidos pelas estações de tratamentos de Barueri e Suzano (ambas no Estado de São Paulo, Brasil) podem conter poluentes orgânicos de importância ambiental. PARAÍBA e SAITO (2005) utilizaram o modelo de fugacidade para simular a distribuição no ar, água, solo, sedimento, plantas e biota aquática de 29 poluentes orgânicos de lodos. Verificaram que em todos os compartimentos ambientais simulados pode ser encontrado pelo menos um desses compostos. KULHANEKA et al. (2005) utilizaram correlações termodinâmicas para determinar o fator de bioconcentração de hidrocarbonetos policíclicos aromáticos em batatas, frutas e hortaliças, cultivadas em solos contaminados com poluentes orgânicos encontrados em lodos.

De forma geral, o fator de bioconcentração (BCF, bioconcentration factor) de um composto num organismo consiste no coeficiente de partição do composto entre o organismo e o meio em que se encontra. No estado de equilíbrio estacionário, esse parâmetro é o quociente entre a concentração no organismo e a concentração no meio. Assim, o valor numérico do BCF indica o grau de partição do poluente entre o organismo e o meio. Quando os organismos são plantas frutíferas, o BCF permite estimar a ingestão diária do poluente pelo consumo diário da fruta quando se conhece a concentração do poluente na solução do solo. Como as frutas são compartimentos finais de várias substâncias absorvidas do solo pela planta, a estimativa do BCF de poluentes em frutas permite também estabelecer limites seguros de poluentes em solos.

Para atender a diretiva 91/414/EEC da Comunidade Econômica Européia, o governo brasileiro está elaborando normas e procedimentos para serem seguidos e aplicados por agências reguladoras, 
produtores e exportadores brasileiros de frutas frescas e de sucos de frutas. A referida diretiva estabelece valores limites de concentrações de compostos orgânicos em produtos agrícolas de origem vegetal (EEC, 1991). Assim, a preocupação do governo brasileiro com a qualidade das frutas com respeito às concentrações de compostos orgânicos está de acordo com as expectativas de brasileiros e de estrangeiros, consumidores de frutas e de sucos de frutas produzidos no Brasil.

O objetivo deste trabalho foi estimar o fator de bioconcentração de dezenove poluentes orgânicos para indicar quais devem ser monitorados em frutos de laranjeiras cultivadas em solos tratados com lodos. Para tanto, foi assumido cultivo hipotético de laranjeiras em solo tratado com lodo e utilizado o modelo Fruit Tree Model para estimar o fator de bioconcentração dos poluentes em frutas.

\section{MATERIAL E MÉTODOS}

O modelo Fruit Tree Model (FTM) de TRAPP, RASMUSSEN e SAMS $\varnothing$ E-PETERSEN (2003), foi utilizado para estimar o BCF de poluentes orgânicos encontrados em amostras de lodos das estações de tratamento do Estado de São Paulo, Brasil. Na Tabela 1 são apresentadas as propriedades físico-químicas dos poluentes necessárias para realizar a estimativa do BCF, utilizando o modelo FTM. No desenvolvimento do modelo FTM foram mantidas as correlações adotadas por TRAPP, RASMUSSEN e SAMS $\varnothing$ E-PETERSEN (2003) para calcular o coeficiente de partição do poluente entre a solução do solo e o carbono orgânico do solo, o fator de bioconcentração do poluente no fluxo de transpiração (TSCF, transpiration stream concentration factor) e o coeficiente de partição do poluente entre o caule e a água. Os 19 poluentes adotados neste estudo foram selecionados pela sua persistência no solo e por constarem na relação de poluentes de lodos publicada por TSUTIYA et al. (2000).

\section{TABELA 1 - NÚMERO DE REGISTRO DO POLUENTE (CAS), MASSA MOLAR, PRESSÃO DE VAPOR, SOLUBILIDADE EM ÁGUA E LOGARITMO DECIMAL DO COEFICIENTE DE PARTIÇÃO OCTANOL-ÁGUA (Kow) DE 19 POLUENTES ORGÂNICOS PRESENTES EM AMOSTRAS DE LODOS DAS ESTAÇÕES DE TRATAMENTO DE ESGOTO DE BARUERI E DE SUZANO (SP)}

\begin{tabular}{lccccc}
\hline \multicolumn{1}{c}{ Poluente } & CAS $^{(1)}$ & $\begin{array}{c}\text { Massa }_{\text {molar }^{(2)}} \\
\left(\mathrm{g} \mathrm{mol}^{-1}\right)\end{array}$ & $\begin{array}{c}\text { Pressão } \\
\text { de vapor } \\
(\mathrm{Pa})\end{array}$ & $\begin{array}{c}\text { Solubilidade } \\
\text { em água }\end{array}$ & LogK $^{(2)}$ \\
& & $(2)$ & $\left(\mathrm{g} \mathrm{m}^{-3}\right)$ & \\
\hline 1,2-diclorobenzeno & $95-50-1$ & 147,00 & $1,81 \times 10^{2}$ & $1,56 \times 10^{2}$ & 3,4 \\
1,3-diclorobenzeno & $541-73-1$ & 147,00 & $2,87 \times 10^{2}$ & $1,25 \times 10^{2}$ & 3,5 \\
1,4-diclorobenzeno & $106-46-7$ & 147,00 & $2,32 \times 10^{2}$ & $8,13 \times 10$ & 3,4 \\
2,4-dinitrofenol & $51-28-5$ & 184,11 & $2,93 \times 10^{-6}$ & $2,79 \times 10^{3}$ & 1,7 \\
3,3-diclorobenzidina & $91-94-1$ & 253,13 & $3,41 \times 10^{-5}$ & 3,10 & 3,5 \\
Antraceno & $120-12-7$ & 178,24 & $3,56 \times 10^{-4}$ & $4,34 \times 10^{-2}$ & 4,5 \\
benzo(a)antraceno & $56-55-3$ & 228,30 & $2,53 \times 10^{-4}$ & $9,40 \times 10^{-3}$ & 5,8 \\
benzo(a)pireno & $50-32-8$ & 252,32 & $7,32 \times 10^{-7}$ & $1,62 \times 10^{-3}$ & 6,1 \\
benzo(k)fluoranteno & $207-08-9$ & 252,32 & $1,29 \times 10^{-7}$ & $8,00 \times 10^{-4}$ & 6,1 \\
dibenzo(a,h)antraceno & $53-70-3$ & 278,36 & $1,33 \times 10^{-8}$ & $2,49 \times 10^{-3}$ & 6,8 \\
fenantreno & $85-01-8$ & 178,24 & $1,49 \times 10^{-2}$ & 1,15 & 4,5 \\
hexaclorobenzeno & $118-74-1$ & 284,78 & $2,40 \times 10^{-3}$ & $6,20 \times 10^{-3}$ & 5,7 \\
hexaclorobutadieno & $87-68-3$ & 260,76 & $2,93 \times 10$ & 3,20 & 4,8 \\
hexacloroetano & $67-72-1$ & 236,74 & $2,80 \times 10$ & $5,00 \times 10$ & 4,1 \\
indeno(1,2,3-c,d)pireno & $193-39-5$ & 276,34 & $1,67 \times 10^{-8}$ & $1,90 \times 10^{-4}$ & 6,7 \\
nitrobenzeno & $98-95-3$ & 123,11 & $1,67 \times 10^{-8}$ & $2,09 \times 10^{3}$ & 1,9 \\
n-nitrosodipropilamina & $10595-95-6$ & 74,08 & $3,60 \times 10^{6}$ & $1,00 \times 10^{6}$ & $-0,6$ \\
pentaclorofenol & $87-86-5$ & 266,34 & $1,47 \times 10^{-2}$ & $1,40 \times 10$ & 5,1 \\
pireno & $129-00-0$ & 202,26 & $6,00 \times 10^{-4}$ & $1,35 \times 10^{-1}$ & 4,9 \\
\hline
\end{tabular}

${ }^{(1)}$ Chemical Abstract Service Registry Number ; (2)Valores fornecidos pela SRC (2005). 
O modelo FTM foi desenvolvido para estimar a concentração de compostos orgânicos nãoiônicos no caule e em frutas, os quais foram absorvidos do solo por plantas frutiferas perenes, podendo ser usado para estimar o BCF de poluentes orgânicos em frutas de frutiferas cultivadas em solos tratados com lodo. No modelo FTM supōe-se que o processo de absorção de poluentes por plantas está em estado de equilibrio estacionário e que as concentrações do poluente no xilema/floema estão em equilibrio quimico em todos os compartimentos da planta. Supöe-se também que os processos de diluição e transformação do poluente, devidos ao crescimento e ao metabolismo da planta, sejam descritos por equaçöes cinéticas de primeira ordem. Como o modelo FTM năo considera as trocas por difusão do poluente no xilema e no floema com o ar ou com a casca do caule, o transporte do poluente na planta consiste em processo passivo que ocorre na transpiraçăo de água absorvida do solo pelas raizes. No modelo FTM, esse processo é descrito pela expressão 1 (TRAPP, RASMUSSEN e SAMS $\varnothing E-P E T E R S E N 2003)$ :

Na qual:

$$
\frac{\mathrm{dl}_{\mathrm{m}, \rho}}{\mathrm{dt}}=Q \mathrm{C}_{\mathrm{Xy}}
$$

$I_{m p}=I_{m p}(t)\left(m g\right.$ ano $\left.{ }^{-1}\right)=$ massa do poluente absorvida pela planta;

$Q\left(L_{\text {ano }}^{-1}\right)=$ volume de água transpirada pela planta; $\mathrm{e}$

$\mathrm{C}_{\mathrm{xy}}\left(\mathrm{mg} \mathrm{L} \mathrm{L}^{-1}\right)=$ concentração do poluente na seiva do xilema.

Segundo TRAPP e MATTHIES (1998), a concentração do poluente na seiva do xilema ( $\mathrm{C}_{x \mathrm{x}}$ ) pode ser estimada a partir da concentração do poluente na solução do solo $\left(C_{w}\right)$ e do valor numérico do TSCF usando-se a expressão 2 :

$$
C_{x y}=\text { TSCFC }_{w}
$$

Na qual:

$\mathrm{C}_{\mathrm{W}}\left(\mathrm{mg} \mathrm{L}^{-1}\right)=$ concentração do poluete na solução do solo; $\mathrm{e}$

TSCF $=$ fator de concentração do poluente no fluxo de transpiração da seiva no xilema.

No modelo FTM, o TSCF é estimado a partir do coeficiente de partição octanol-água $\left(K_{\text {ow }}\right)$ por meio da correlação proposta por BURKEN e SCHNOOR (1998) que é dada por:

Na qual:

$$
\text { TSCF }=0,756 \exp \left[\frac{-\left(\log _{\mathrm{ow}}-2,50\right)^{2}}{2,58}\right]
$$

Log $\mathrm{K}_{\mathrm{ow}}=$ logaritmo decimal do coeficiente de partiçăo do poluente entre o octanol e a água.

Define-se o TSCF como o quociente entre a concentraçāo do composto orgânico no fluxo de transpiração e sua concentraçăo na solução do solo (TRAPP e MATTHIES, 1998). Por meio do fluxo de água no caule, o poluente é transportado para as folhas e frutas. No caule o poluente é transformado por processos metabólicos, sendo diluído pelo crescimento da planta. Essa etapa é descrita no modelo FTM pela equaçăo 4 (TRAPP, RASMUSSEN e SAMS $\varnothing E-P E T E R S E N, 2003$ ):

Na qual:

$$
\frac{d E_{m, D}}{d t}=\left(k_{E}+k_{G}\right) M_{C L} C_{C L}+\frac{Q C_{C L}}{k_{C L, W}}
$$

$E_{m, p}=E_{m p}(t)(m g$ ano-1) = massa do poluente no caule ao longo do tempo;

$M_{\mathrm{cL}}(\mathrm{kg})=$ massa total seca de caule;

$k_{E}\left(a^{-1}\right)=$ taxa de transformaçăo do poluente no caule;

$\mathrm{k}_{\mathrm{o}}\left(\mathrm{ano}^{-1}\right)=$ taxa anual de crescimento da massa de caule;

$\mathrm{C}_{\mathrm{cL}}\left(\mathrm{mg} \mathrm{kg}^{-1}\right)=$ concentração do poluente no caule; $\mathrm{e}$

$\mathrm{K}_{\text {c.w }}\left(\mathrm{L} \mathrm{kg}^{-1}\right)=$ coeficiente de partição do poluente entre o caule e a água.

$O$ valor do $\mathrm{K}_{\mathrm{cLw}}$ foi estimado a partir do coeficiente de partição octanol-água do poluente por meio da correlaçăo proposta por TRAPP, MIGLIORANZ e MOSBAEK (2001) que é dada por:

$$
K_{\mathrm{CLLW}}=10^{\left(-0,27 * 0,063 \times \log K_{\mathrm{ow}}\right)}
$$


Como o fluxo de água no caule transporta o poluente para as folhas e frutas, o balanço total de massa do poluente na planta pelo modelo FTM é calculado pela equaçăo 6 :

Na qual:

$$
\frac{d m_{p}}{d t}=\frac{d I_{m, p}}{d t} \cdot \frac{d E_{m p p}}{d t}
$$

$\mathrm{m}_{\mathrm{p}}=\mathrm{m}_{\mathrm{p}}(\mathrm{t})\left(\mathrm{mg} \mathrm{ano}^{-1}\right)=$ massa do poluente na planta ao longo do tempo.

Supondo que $m_{p}(0)=0$ a soluçăo da equação $(6)$ é dada por:

$$
m_{p}(t)=\frac{A}{B}(1-\exp (-B t))
$$

Em que as constantes $\mathrm{A}\left(\mathrm{mg} \mathrm{kg}^{-1} \mathrm{ano}^{-1}\right)$ e $\mathrm{B}\left(\mathrm{ano}^{-1}\right)$ săo definidas por:

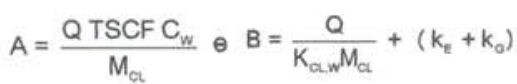

A constante $\mathrm{A}=$ taxa de transferência do composto da solução do solo para a planta por meio da transpiração; e a constante $B$ = taxa de dissipação e de sorção do poluente na planta, denominada de taxa de transformaçāo do poluente na planta.

Fazendo-se $m_{p}(t)=M_{C L} C_{C L}(t)$ na equação (7), a soluçăo em estado estacionário que descreve a concentraçăo em equilibrio estável é dada por:

$$
C_{a L}=\frac{Q \operatorname{TSCFC}_{w}}{\frac{Q}{K_{\text {ouw }}}+M_{\mathrm{a}}\left(k_{\mathrm{E}}+k_{0}\right)}
$$

A concentração do poluente na solução do solo pode ser estimada a partir da concentração do poluente no solo úmido, $\mathrm{C}_{\mathrm{s}}\left(\mathrm{mg} \mathrm{kg}^{-1}\right)$, pela expressåo 9 (TRAPP e MATTHIES, 1998):

$\mathrm{Na}$ qual:

$$
C_{w}=\frac{\rho_{-} C_{5}}{\rho_{5} f_{O c} K_{O C}+f_{w}+f_{A} K_{A w}}
$$

$\rho_{\mathrm{w}}\left(\mathrm{kg} \mathrm{L}^{-1}\right)$ e $\rho_{\mathrm{s}}\left(\mathrm{kg} \mathrm{L}^{-1}\right)=$ densidades do solo úmido e do solo seco, respectivamente;

Os coeficientes $f_{o c}, f_{w}$ e $f_{A}=$ fraç̋̋es volumétricas de carbono orgânico, água e ar do solo, respectivamente;

O parâmetro $\mathrm{K}_{\mathrm{oc}}\left(\mathrm{L} \mathrm{kg}^{-1}\right)=$ coeficiente de partiçăo do poluente entre o carbono orgânico; $\Theta$

A soluçăo no solo e $\mathrm{K}_{\mathrm{AW}}$ = coeficiente de partiçăo do poluente entre 0 ar e a água.

O coeficiente de partiçăo do poluente entre o carbono orgânico e a soluçăo do solo $\left(\mathrm{L} \mathrm{kg}^{-1}\right)$ foi estimado, segundo recomendação de TRAPP, RASMUSSEN e SAMS $\varnothing E-P E T E R S E N(2003)$, usandose o coeficiente de partiçăo octanol-água do poluente pela expressăo 10 (BEELEN, 2000):

$$
\mathrm{K}_{\mathrm{oC}}=10^{\left(0,81 \times \log _{\mathrm{ow}}+0,1\right)}
$$

O coeficiente de partiçăo entre o ar e a água foi estimado pela expressão 11 (TRAPP e MATTHIES, 1998):

$$
K_{\text {Aw }}=\frac{P_{v} m_{M}}{(273+T) R w_{s}}
$$

Na qual:

$\mathrm{T}\left(=25^{\circ} \mathrm{C}\right)=$ temperatura do $\mathrm{ar}$;

$\mathrm{R}\left(=8,314 \mathrm{~Pa} \mathrm{~m}^{3} \mathrm{~mol}^{-1} \mathrm{~T}^{-1}\right)=$ constante do gases;

$\mathrm{P}_{\mathrm{v}}(\mathrm{Pa}), \mathrm{m}_{\mathrm{M}}\left(\mathrm{g} \mathrm{mol}^{-1}\right)$ e $\mathrm{W}_{\mathrm{S}}\left(\mathrm{g} \mathrm{m}^{-3}\right)=$ massa molar, pressāo de vapor e solubilidade em água do poluente, respectivamente.

Segundo TRAPP, RASMUSSEN e SAMS $\varnothing E-P E T E R S E N ~(2003)$, a acumulação do poluente em frutas pode ocorrer pelo xilema e pelo floema, que repōem a água transpirada pela casca dos 
frutos e proporcionam seu desenvolvimento. Assim, a massa de água necessária para a formação de frutas frescas pode ser estimada pela equaçăo 12 (TRAPP, RASMUSSEN e SAMS $\varnothing E-P E T E R S E N$, 2003):

Na qual:

$$
Q_{F L}=20 d_{w}
$$

$Q_{\mathrm{FL}}\left(\mathrm{L} \mathrm{kg}^{-1}\right)=$ volume necessário de água em litros para a produção de um quilo de fruta fresca; $\mathrm{e}$ $\mathrm{d}_{\mathrm{w}}=$ fraçăo de matéria seca da fruta. Para os frutos de laranjeira foram considerados $20 \%$ de matéria $\operatorname{seca}\left(d_{w}=0,2\right)$.

Assumindo equilibrio quimico entre caule, xilema e floema, a concentraçăo do poluente na fruta pode ser calculada pela expressåo 13:

$$
\mathrm{C}_{\mathrm{rT}}=\frac{\mathrm{Q}_{\mathrm{rL}} \mathrm{C}_{\mathrm{CL}}}{\mathrm{K}_{\mathrm{ch}, \mathrm{w}}}
$$

Na qual:

$\mathrm{C}_{\mathrm{FT}}\left(\mathrm{mg} \mathrm{kg}^{-1}\right)=$ concentração do poluente na fruta fresca.

O fator de bioconcentração do poluente na fruta foi obtido pelo quociente entre a concentraçăo do poluente na fruta e a concentraçăo do poluente na solução do solo, dado pela equaçăo 14 :

$$
B C F=\frac{C_{F T}}{C_{W}}=\frac{Q_{F L} Q T S C F}{Q+K_{C L W} M_{C L}\left(k_{E}+k_{\odot}\right)}
$$

Na qual:

$B C F\left(\mathrm{~L} \mathrm{~kg}^{-1}\right)=$ fator de bioconcentração do poluente na fruta.

Assumiu-se cultivo de plantas adultas de laranjeiras com evapotranspiraçäo de $7,5 \times 10^{6} \mathrm{~L}$ $\mathrm{ha}^{-1} \mathrm{ano}^{-1}\left(750 \mathrm{~mm}\right.$ ano-1) , biomassa em peso seco de $10^{4} \mathrm{~kg} \mathrm{ha}^{-1}$, correspondendo ao plantio de 400 plantas por hectare com peso médio seco de caule de $25 \mathrm{~kg}_{\text {planta-1 }}{ }^{-1}$ taxa de crescimento de 0,01 por ano. A taxa de metabolismo do poluente na planta de $3,69 \mathrm{ano}^{-1}\left(\mathrm{k}_{\mathrm{E}}\right)$ foi estimada a partir de valores da meia-vida de compostos orgânicos em plantas, obtidos em COUSINS e MACKAY (2001). Considerouse solo com 1,79 $\mathrm{kg} \mathrm{L}^{-1}$ de densidade do solo úmido, $1,25 \mathrm{~kg} \mathrm{~L}^{-1}$ de densidade do solo seco, 0,016 de conteúdo volumétrico de carbono orgânico, 0,3 de conteúdo volumétrico de água e 0,24 de conteúdo volumétrico de ar.

O fator de concentração na raiz (RCF, root concentration factor) de BRIGGS, BROMILOW e EVANS (1982) constitui indicador da bioconcentração potencial de um composto orgânico em raizes de plantas. Segundo BRIGGS, BROMILOW e EVANS (1982), o valor do RCF está correlacionado com o coeficiente de partiçăo octanol-água pela expressão $R C F=10^{\left(-1.52-a . m l_{00} K_{m}\right)}+0,82$. Define-se o RCF como o quociente entre a concentraçăo na raiz e a concentraçăo na solução do solo determinadas em sistema em equilíbrio químico contendo o composto orgânico, a raiz e a soluçăo do solo. Usou-se o RCF para analisar os valores do fator de bioconcentração dos poluentes em frutos de laranjeira estimados pelo modelo FTM.

A massa molecular, a pressão de vapor, a solubilidade em água e o coeficiente de partição octanol-água de cada poluente (Tabela 1) foram obtidos da Syracuse Research Corporation (SRC, 2005).

\section{RESULTADOS E DISCUSSÃO}

Pode-se observar pela Tabela 1 que dezesseis dos dezenove poluentes estudados têm valores de $\mathrm{LogK}_{\mathrm{ow}}>3$ ou $\mathrm{K}_{\mathrm{ow}}>1000$, o que resulta em expressiva afinidade desse conjunto de poluentes ao carbono orgânico presente no solo e nas plantas. Esses dezesseis poluentes seriam absorvidos $e$ preferencialmente encontrados na matéria orgânica do solo ou nas raizes das plantas (PARAíBAe SAITO, 2005)

O coeficiente de partiçăo octanol-água da água tem valor 0,042 ( LogKow $=-1,38$ ) (SRC, 
2005), o que acarreta RCF de valor 1,0 para a água. Considerando-se a água como substância de referência para a translocaçăo de substâncias do solo para as frutas espera-se que os poluentes com valores de RCF próximos de 1,0 sejam absorvidos pela planta e que apresentem valores significativos de BCF em frutas (Tabela 2). Quanto maior o RCF do poluente em relaçăo ao RCF da água, menor será o seu BCF em frutas (Tabela 2).

\section{TABELA 2 - COEFICIENTE DE PARTIÇÃO EM RELAÇÃO AO TEOR DE CARBONO ORGÂNICO DO SOLO ( $\left.K_{\propto}\right)$, COEFICIENTE DE PARTIÇĂO AR-ÁGUA $\left(K_{\text {Aw }}\right)$, COEFICIENTE DE PARTIÇĀO CAULE-ÁGUA (K $\left.\mathrm{K}_{\mathrm{CL}, W}\right)$, FATOR DE CONCENTRAÇĀO NO FLUXO TRANSPIRADO (TSCF), FATOR DE CONCENTRAÇĀO NA RAIZ (RCF) E FATOR DE BIOCONCENTRAÇÃO (BCF) EM FRUTOS DE LARANJEIRAS DE 19 POLUENTES ORGÂNICOS DE LODOS DE ESGOTOS}

\begin{tabular}{lcccccc}
\hline \multicolumn{1}{c}{ Poluente } & $\mathrm{KoC}$ & $\mathrm{K}$ AW & $\mathrm{K} \mathrm{CL}, \mathrm{W}$ & TSCF & RCF & BCF \\
& $\left(\mathrm{L} \mathrm{kg}^{-1}\right)$ & - & $\left(\mathrm{L} \mathrm{kg}^{-1}\right)$ & - & - & $\left(\mathrm{L} \mathrm{kg}^{-1}\right)$ \\
\hline 1,2-diclorobenzeno & 756 & $6,88 \times 10^{-2}$ & 80 & 0,541 & 14 & 1,9400 \\
1,3-diclorobenzeno & 911 & $1,36 \times 10^{-1}$ & 92 & 0,501 & 17 & 1,7219 \\
1,4-diclorobenzeno & 770 & $1,69 \times 10^{-1}$ & 81 & 0,537 & 14 & 1,9181 \\
2,4-dinitrofenol & 28 & $7,79 \times 10^{-11}$ & 6 & 0,579 & 1 & 2,8089 \\
3,3-diclorobenzidina & 877 & $1,12 \times 10^{-6}$ & 90 & 0,509 & 16 & 1,7652 \\
antraceno & 5064 & $5,90 \times 10^{-4}$ & 352 & 0,173 & 81 & 0,3164 \\
benzo(a)antraceno & 58291 & $2,48 \times 10^{-3}$ & 2368 & 0,012 & 823 & 0,0048 \\
benzo(a)pireno & 116225 & $4,60 \times 10^{-5}$ & 4057 & 0,005 & 1586 & 0,0011 \\
benzo(k)fluoranteno & 111970 & $1,64 \times 10^{-5}$ & 3940 & 0,005 & 1531 & 0,0012 \\
dibenzo(a,h)antraceno & 369403 & $6,00 \times 10^{-7}$ & 10000 & 0,001 & 4760 & 0,0001 \\
fenantreno & 5159 & $9,32 \times 10^{-4}$ & 357 & 0,171 & 83 & 0,3088 \\
hexaclorobenzeno & 55119 & $4,45 \times 10^{-2}$ & 2267 & 0,013 & 781 & 0,0054 \\
hexaclorobutadieno & 9371 & $9,64 \times 10^{-1}$ & 569 & 0,101 & 146 & 0,1324 \\
hexacloroetano & 2841 & $5,35 \times 10^{-2}$ & 224 & 0,267 & 47 & 0,6329 \\
indeno(1,2,3-c,d)pireno & 336512 & $9,80 \times 10^{-6}$ & 9298 & 0,001 & 4356 & 0,0001 \\
nitrobenzeno & 40 & $3,97 \times 10^{-13}$ & 8 & 0,642 & 2 & 3,0871 \\
n-nitrosodipropilamina & 0,4348 & $1,08 \times 10^{-1}$ & 0,5 & 0,020 & 1 & 0,0978 \\
pentaclorofenol & 17669 & $1,13 \times 10^{-4}$ & 933 & 0,053 & 265 & 0,0472 \\
pireno & 11293 & $3,63 \times 10^{-4}$ & 658 & 0,084 & 174 & 0,0991 \\
\hline
\end{tabular}

Segundo TRAPP, RASMUSSEN e SAMS $\varnothing E-P E T E R S E N ~(2003)$, compostos com TSCF próximos de 0,393 ( $\left.\operatorname{LogK}_{\text {ow }} \cong 1,2\right)$ apresentam condiçōes ótimas para transferência da solução do solo para as frutas. Pelos dados da Tabela 2 verifica-se que o 2,4-dinitrofenol ( $\left.\operatorname{LogK}_{\mathrm{ow}}=1,7\right)$ e o nitrobenzeno ( $\log \mathrm{K}_{\mathrm{ow}}=1,9$ ) revelaram os menores RCF e os maiores TSCF (Tabela 2). Considerando o conjunto dos fatores RCF, TSCF e BCF, o 1,2-diclorobenzeno, o 1,3-diclorobenzeno, o 1,4diclorobenzeno, 0 2,4-dinitrofenol, o 3,3-diclorobenzidina e o nitrobenzeno deveriam ser monitorados prioritariamente em frutos de laranjeiras cultivadas em solos abonados com lodo de esgoto provenientes de estaçőes de tratamento do Estado de Săo Paulo.

O valor do BCF na fruta permite estimar a ingestão diária de um poluente por peso corpóreo, devido ao consumo de laranjas cultivadas em solos que receberam lodo como fertilizante. Permite também estabelecer limites aceitáveis para o uso agrícola de lodos que contenham poluentes orgânicos. Supondo-se, por exemplo, que o solo usado neste trabalho fosse fertilizado com a dose de $10 \mathrm{~T} \mathrm{ha}^{-1} \mathrm{de}$ lodo contendo 2,4-dinitrofenol na concentração de 4,8 $\mathrm{mg} \mathrm{kg}^{-1}$ em base seca. Esses valores proporcionariam da superficie até a profundidade de 0,2 m concentraçōes de 2,4-dinitrofenol de 2,68x 
$10^{-2} \mathrm{mg} \mathrm{kg}^{-1}$ no solo e de $5,54 \times 10^{-2} \mathrm{mg} \mathrm{L}^{-1}$ na soluçăo do solo. Essas concentraçöes, juntamente com o BCF de 2,4-dinitrofenol de 2,81 $\mathrm{L} \mathrm{kg}^{-1}$ (Tabela 2), acarretariam concentração em frutos de laranjeira de $1,55 \times 10^{-1} \mathrm{mg} \mathrm{kg}^{-1}$ e ingestão diária de $1,11 \times 10^{-3} \mathrm{mg} \mathrm{kg}^{-1}$ de 2,4-dinitrofenol por peso corpóreo. Tal ingestāo diária foi estimada para indivíduo com $70 \mathrm{~kg}$ que consumisse diariamente $0,5 \mathrm{~kg}$ de laranja fresca. Sua ordem de grandeza alcançaria o valor da dose de referência (RfD, reference dose) de

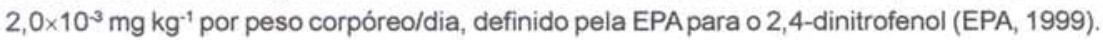

Em termos gerais, a RfD estima a exposição diária da população humana ao agente químico que năo causaria risco associado aos efeitos deletérios ao longo da vida. O valor da RfD é expresso em miligrama do agente químico por quilograma de peso corpóreo por dia ( $\left.\mathrm{mg} \mathrm{kg}^{-1} \mathrm{dia}^{-1}\right)$ (EPA, 1993). Assim, os frutos de plantas cultivadas em solos fertilizados com lodo deveriam ser monitorados quanto as concentrações de 2,4-dinitrofenol ou, ainda, doses maiores do que $10 \mathrm{~T}^{\text {ha-1 }}{ }^{-1}$ desse tipo de lodo não deveriam ser usadas para o cultivo de frutiferas. 02,4 -dinitrofenol (DNP) é empregado para fabricar tintas, preservativos para madeira, explosivos, inseticidas e reveladores fotográficos, entre outros. Esse poluente causou aberraçōes cromossômicas em células da linhagem $\mathrm{CHO}$ (chinese hamster ovary) de roedores (HILLIARD et al., 1998).

No desenvolvimento do modelo FTM, TRAPP, RASMUSSEN e SAMS $\varnothing E-P E T E R S E N ~(2003)$ supuseram massa de $1,0 \mathrm{~kg}$ de fruto fresco, concentração de $1,0 \mathrm{mg} \mathrm{kg}^{-1}$ do poluente no solo $\mathrm{e}$ estimaram o BCF do poluente em frutos com unidade de $\mathrm{mg} \mathrm{kg}^{-1}$. A equação 14 , desenvolvida neste trabalho a partir do modelo FTM, generaliza o cálculo do BCF de poluentes em frutos para qualquer massa de fruta fresca e qualquer concentraçăo do poluente no solo. Também apresenta e estima o valor do BCF em L kg-1 (unidade correta para o BCF), o qual é definido como o quociente entre a concentraçăo do poluente na fruta $\left(\mathrm{mg} \mathrm{kg}^{-1}\right)$ e a concentraçăo do poluente na soluçăo do solo $\left(\mathrm{mg} \mathrm{L}^{-1}\right)$. Pela equação 14 pode-se observar que o fator de bioconcentração de poluentes em frutos é diretamente proporcional ao volume de água transpirada pela planta e ao fator de concentraçăo no fluxo de transpiração. Também se pode observar que o fator de bioconcentração é inversamente proporcional à massa de caule, ao coeficiente de partiçăo entre o caule e a água e a taxa de transformaçăo do poluente no caule.

TRAPP, RASMUSSEN e SAMS $\varnothing E-P E T E R S E N ~(2003)$ analisaram a sensibilidade do modelo FTM e constataram que o mesmo é robusto em relaçăo à taxa de transpiração, taxa de crescimento das plantas e em relação à biomassa de caule. Também afirmaram que as incertezas nos valores dessas variáveis não têm influencia significativa no valor do BCF de poluentes em frutas que apresentem $0,8<\log _{\text {ow }}<2,0$, faixa de valores em que se encontram o 2,4-dinitrofenol e o nitrobenzeno. Tais autores supōem que as incertezas nos valores de BCF podem estar associadas aos processos de metabolismo do poluente na planta e à volatilização do poluente no solo ou na planta.

Não se encontrou na literatura valores de BCF de poluentes determinados experimentalmente em frutos de laranjeiras. Os valores de BCF de benzo(a)pireno determinados experimentalmente em frutos por TRAPP, RASMUSSEN e SAMS $\varnothing E-P E T E R S E N$ (2003) eståo na mesma ordem de grandeza do valor do BCF de benzo(a)pireno estimado pela equação 14 e apresentado na Tabela 2.

\section{CONCLUSÃO}

O valor do fator de bioconcentração em frutos de laranjeiras de dezenove poluentes orgânicos

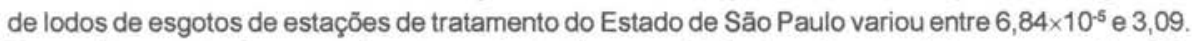
No monitoramento desses poluentes em frutos deve ser dada atenção especial aos compostos 1,2diclorobenzeno, 1,3-diclorobenzeno, 1,4-diclorobenzeno, 2,4-dinitrofenol, 3,3-diclorobenzidina e nitrobenzeno, pois o modelo FTM indicou que esses compostos apresentam os maiores valores de $\mathrm{BCF}$ em frutos de laranjeiras. A estimativa do valor da ingestão diária de poluentes orgânicos de lodos, mediante consumo de frutos frescos cultivados em solos condicionados com lodo de esgoto, pode ser realizada a partir do valor do BCF desses poluentes nos frutos. 


\begin{abstract}
BIOCONCENTRATION FACTOR OF ORGANIC POLLUTANTS OF THE SEWAGE SLUDGE IN ORANGE FRUITS

The bioconcentration factors of nineteen organic pollutants was estimated to indicate which pollutants should be monitored in orange orchards, cultivated in soils treated with sludge originated from sewage treatment plants. The Fruit Tree Model (FTM) was applied in a hypothetical culture of orange orchard by using molar mass, vapor pressure, water solubility and the octanol-water partition coefficient (non ionic organic compounds) that can be found in sludge. The soil organic carbon sorption coefficient, stem-water partition coefficient and transpiration stream concentration factor were calculated through equations that correlate each variable with the octanol-water partition coefficient. Once the bioconcentration factor and soil pollutant concentration are known, it is possible to estimate the chemical agent daily intake by fresh fruit consumption. Sewage sludge pollutant limit values may be established from the pollutant bioconcentration factor values determined in fruits. The simulation indicated that the following pollutants should be monitored in orange orchards treated with sewage sludge: 1,2-dichlorobenzene, 1,3- dichlorobenzene, 1,4- dichlorobenzene, 2,4-dinitrophenol, 3,3dichlorobenzidine and nitrobenzene.
\end{abstract}

KEY-WORDS: FERTILIZER; CITRUS; DAILY INTAKE; BIOSOLID; SEWAGE SLUDGE.

\title{
REFERÊNCIAS
}

ABAD, E.; MARTINEZ, K.; PLANAS, C.; PALACIOS, O.; CAIXACH, J.; RIVERA, J. Priority organic pollutant assessment of sludges for agricultural purposes. Chemosphere, v.61, n.9, p.1358-1369, 2005.

BEELEN, P. Van. The risk evaluation of difficult substances in USES 2.0 and EUSES: a decision tree for data gap filling of Kow, Koc, and BCF. Bilthoven: Rijksinstituut voor Volksgezondheid en Milieu, 2000. 35 p. (RIVM Report 679102050).

BETTIOL, W.; SANTOS, I. Efeito do lodo de esgoto em fitopatógenos veiculados pelo solo. Jaguariúna: Embrapa Meio Ambiente, 2001. 30 p. (Embrapa Meio Ambiente. Documentos, 24).

BOEIRA, R.C.; LIGO, M.A.V.; DYNIA, J.F. Mineralização de nitrogênio em solo tropical tratado com lodos de esgoto. Pesquisa Agropecuária Brasileira, v.37, p.1639-1647, 2002.

BOYD, G.R.; REEMTSMA, H.; GRIMM, D.A.; MITRA, S. Pharmaceuticals and personal care products (PPCPs) in surface and treated waters of Louisiana, USA and Ontario, Canada. Science of the Total Environment, v.311, n.1-3, p.135-149, 2003.

BRIGGS, G.G.; BROMILOW, R.H.; EVANS, A.A. Relationships between lipophilicity and root uptake and translocation of no ionised chemicals by barley. Pesticide Science, v.13, p.495-504, 1982.

BURKEN, J.G.; SCHNOOR, J.L. Predictive relationships for uptake of organic contaminants by hybrid poplar trees. Environmental Science and Technology, v.32, n.21, p.3379-3385, 1998.

CARBALLA, M.; OMIL, F.; LEMA, J.M.; LLOMPART, M.; GARCIA-JARES, C.; RODRIGUEZ, I.; GOMEZ, M.; TERNES, T. Behavior of pharmaceuticals, cosmetics and hormones in a sewage treatment plant. Water Research, v.38, p.12, p.2918-2926, 2004.

CARBALLA, M.; OMIL, F.; LEMA, J.M.; LLOMPART, M.; GARCIA, C.; RODRIGUEZ, I.; GOMEZ, M.; TERNES, T. Behaviour of pharmaceuticals and personal care products in a sewage treatment plant of northwest Spain. Water Science and Technology, v.52, n.8, p.29-35, 2005.

CARGOUET, M.; PERDIZ, D.; MOUATASSIM-SOUALI, A.; TAMISIER-KAROLAK, S.; LEVI, Y. Assessment of river contamination by estrogenic compounds in Paris area (France). Science of the Total Environment, v.324, n.1-3, p.55-66, 2004.

COUSINS, I. T.; MACKAY, D. Strategies for including vegetation compartments in multimedia models. Chemosphere, v.44, p. 643-654, 2001.

EEC. European Economic Community. Council Directive 91/414/EEC. Concerning the placing of plant protection products on the market. Office for Official Publications of the European Communities. Official Journal of the European Union, 1991, 194 p. Disponível em: <http://europa.eu.int/eur- lex/en/consleg/pdf/1991/ en_1991L0414 _do_ 001.pdf>. Acesso em: 2 jan. 2006.

ENGWALL, M.; HJELM, K. Uptake of dioxin-like compounds from sewage sludge into various plant species assessment of levels using a sensitive bioassay. Chemosphere, v.40, n.9-11, p.1189-1195, 2000. 
EPA. Environmental Protection Agency. Integrated Risk Information System (IRIS) on 2,4-dinitrophenol. Washington: National Center for Environmental Assessment, Office of Research and Development, 1999. Disponível em: <http://www.epa.gov/iris/subst/0152.htm>. Acesso em: 21 fev.2005.

EPA. Environmental Protection Agency. Integrated Risk Information System. Reference Dose (RfD): description and use in health risk assessments. Background Document 1A March 15, 1993. Disponível em: www.epa.gov/ iris/rfd.htm. Acessado em: 07 de fev.2006

GAO, Y.; ZHU, L. Plant uptake, accumulation and translocation of phenanthrene and pyrene in soils. Chemosphere, v.55, p.1169-1178, 2004.

HILLIARD, C.A.; ARMSTRON, M.J.; BRADT, C.I.; HILL, R.B.; GREENWOOD, S.K.; GALLOWAY, S.M. Chromosome aberrations in vitro related to cytotoxicity of nonmutagenic chemicals and metabolic poisons. Environmental and Molecular Mutagenesis, v.31, n.4, p.316-326, 1998.

KULHANEKA, A.; TRAPP, S.; SISMILICH, M.; JANKU, J.; ZIMOVA, M. Crop-specific human exposure assessment for polycyclic aromatic hydrocarbons in Czech soils. Science of the Total Environment, v.339, v.1-3, p.7180,2005

MADEJON, E.; BURGOS, P., LÓPEZ, R.; CABRERA, F. Agricultural use of three organic residues: effect on orange production and on properties of a soil of the "Comarca Costa de Huelva" (SW Spain). Nutrient Cycling in Agroecosystems, v.65, n.3, p.281 - 288, 2003.

MIADOKOVA, E.; DUHOVA,V.; VLCKOVA, V.; SLADKOVA, L.; SUCHA, V.; VLCEK, D. Genetic risk assessment of acid waste water containing heavy metals. General Physiology and Biophysics, v.18, p.92-98, 1999.

NEDELCHEVA,V.; GUT, I.; SOUCEK, P.; FRANTIK, E. Cytochrome P450 catalyzed oxidation of monochlorobenzene, 1,2- and 1,4-dichlorobenzene in rat, mouse, and human liver microsomes. Chemico-Biological Interactions, v.115, n.1, p.53-70, 1998

PARAÍBA, L.C.; SAITO, M.L. Distribuição ambiental de poluentes orgânicos encontrados em lodos de esgotos. Pesquisa Agropecuária Brasileira, v.40, n.9, p.853-860, 2005.

SRC. Syracuse Research Corporation. Interactive PhysProp database demo. Disponível em: <http:// www.syrres.com/esc/physdemo.htm> Acesso em: 21 fev. 2005.

TERNES, T.A. Occurrence of drugs in German sewage treatment plants and rivers. Water Research, v.32, n.11, p.3245-3260, 1998.

TERNES, T.A.; STUMPF, M.; J. MUELLER, J.; HABERER, K.; WILKEN, R.D.; SERVOS, M. Behavior and occurrence of estrogens in municipal sewage treatment plants: I. Investigations in Germany, Canada and Brazil. The Science of the Total Environment, v.225, p.81-80, 1999.

TRAPP, S.; MATTHIES, M. Chemodynamics and environmental modelling. Heidelberg: Springer, 1998. $285 \mathrm{p}$.

TRAPP, S.; MIGLIORANZA, K.S.B.; MOSBAEK, H. Sorption of lipophilic organic compounds to wood and implications for their environmental fate. Environmental Science \& Technology, v.35, n.8, p.1561-1566, 2001.

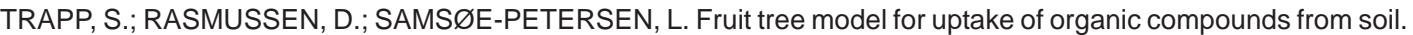
Sar and Qsar in Environmental Research, v.14, n.1, p.17-26, 2003.

TSUTIYA, M.T. Características de biossólidos gerados em estações de tratamento de esgotos. In: TSUTIYA, M.T.; COMPARINI, J.B.; PEREIRA SOBRINHO, A.; HESPANOL, I.; CARVALHO, P.C.T.; MELFI, A.J. (Ed.). Biossólidos na agricultura. São Paulo: SABESP, 2001. Cap.4, p.89-131. 